\title{
EMBARAZO PRECOZ EN ADOLESCENTES DE SECUNDARIA DEL CENTRO EDUCATIVO ESTATAL “MARISCAL CASTILLA”, EL TAMBO, HUANCAYO, 2004
}

\author{
EARLY PREGNANCY IN ADOLESCENTS OF HIGH SCHOOL OF THE STATAL \\ CENTER EDUCATIONAL “MARISCAL CASTILLA”, EL TAMBO, HUANCAYO, 2004
}

\author{
Magda Paredes Caballero' Marivel Martínez Veliz ${ }^{2}$ Julio Soto Rojas ${ }^{2}$
}

\begin{abstract}
RESUMEN
En los últimos diez a quince años ha aumentado significativamente la preocupación de diversos sectores sociales en el departamento de Junín por el fenómeno del embarazo adolescente, de ahí la inquietud de la investigación con el objetivo de obtener información sobre el problema del embarazo precoz en estudiantes adolescentes de secundaria del centro educativo estatal "Mariscal Castilla", del distrito de El Tambo, provincia de Huancayo, durante el año 2004; y dar propuestas con la intención de contribuir a definir con mayor rigor y pertinencia los problemas reales vinculados con el embarazo en adolescentes. La investigación fue descriptiva, transversal, primaria y cuantitativa; la población constituido por las alumnas del $3^{\circ}, 4^{\circ}$ y $5^{\circ}$ grados de secundaria. El uso de métodos anticonceptivos para las adolescentes oscila del $7 \%$ al $30 \%$; con alto porcentaje la práctica el método del ritmo y el retiro (Coitus interruptus), no obstante, desconocen la fisiología del ciclo femenino. El inicio de la actividad sexual antes de los 18 años es una decisión sobre la cual influyen e interactúan una serie de factores.
\end{abstract}

Palabras clave: Aborto, relaciones prematrimoniales, embarazo adolescente.

\begin{abstract}
In the last ones ten to fifteen years have increased the concern of diverse social sectors significantly in the department of Junín for the phenomenon of the adolescent pregnancy, of there the restlessness of the investigation with the objective of obtaining information on the problem of the precocious pregnancy in adolescent students of secondary of the center educational state "Mariscal Castilla", of the district of El Tambo, country of Huancayo, during the year 2004; and to give proposals with the intention of contributing to define with bigger rigor and relevancy the real problems linked with the pregnancy in adolescents. The investigation was descriptive, traverse, primary and quantitative; the population constituted by the students of the $3^{\circ}, 4^{\circ}$ and $5^{\circ}$ grades of secondary. The use of birthcontrol methods for the adolescents oscillates from $7 \%$ to $30 \%$; with high percentage, the practice the method of the rhythm and the retirement (Coitus interruptus), nevertheless, they ignore the physiology of the feminine cycle. The beginning of the sexual activity before the 18 years is a decision on which you/they influence and interaction a series of factors.
\end{abstract}

Key words: Abortion, relationships marriage previous, adolescent pregnancy. 


\section{INTRODUCCIÓN}

En los últimos diez a quince años ha aumentado significativamente la preocupación de diversos sectores sociales en el Perú por el fenómeno del embarazo adolescente, lo cual se ha traducido en políticas y programas de población de salud, de educación, sociales, en los cuáles tienden a invertirse recursos humanos y económicos. Antes de realizar la investigación se tuvo referencia que en los últimos años se viene incrementando los índices de embarazos en las adolescentes del "Colegio Castilla".

El problema planteado fue la siguiente pregunta: ¿cuáles son las causas que originan el embarazo a temprana edad en los adolescentes del Colegio Estatal Mariscal Castilla del distrito de El Tambo, Huancayo durante el año 2004?; como objetivo: determinar las causas que originan el embarazo precoz a temprana edad en las adolescentes, sensibilizar a la población estudiantil del colegio mencionado sobre los efectos negativos en la salud reproductiva y buscar las medidas correctivas para disminuir el embarazo precoz en las adolescentes. Los resultados se deben considerar para ser incluidos en las políticas de gestión del sector educación

\section{MATERIAL Y MÉTODOS}

El área de estudio fue el Centro Educativo Estatal Mariscal Castilla del distrito de El Tambo, provincia de Huancayo. Como universo del estudio se consideró todas las estudiantes de tercero, cuarto y quito años de secundaria. La recolección de datos se realizó en 28 aulas de clases, solamente a estudiantes mujeres. Se aplicaron las encuestas en forma anónima. Para el análisis cuantitativo se utilizó las técnicas estadísticas como la determinación de frecuencias y porcentajes, adicionalmente se empleó técnicas de la estadística inferencial.

\section{RESULTADOS}

El medio que reciben orientación sexual las

Tabla 1. Recibió orientación sexual.

\begin{tabular}{lc}
\hline Medio & N $^{\circ}$ de alumnas \\
\hline Salud & 30 \\
Colegio - familiar & 115 \\
Colegio & 215 \\
Padres & 100 \\
Autoeducación & 30 \\
No recibió & 80 \\
\hline
\end{tabular}

adolescentes encuestadas en su mayor porcentaje es en el colegio, continuado por el colegio y familiares, padres y en mínimo porcentaje del sector salud., evidenciando que no existe una buena comunicación entre padres e hijas, notándose también el poco trabajo del sector salud,

El uso de métodos anticonceptivos para las adolescentes oscila entre un 7 y $30 \%$. De estos, el $75 \%$ practica el método del ritmo y el retiro (coitus interruptus) pero desconocen la fisiología del ciclo femenino.

Tabla 2. Ha iniciado relaciones sexuales.

\begin{tabular}{cc}
\hline Respuesta: & $\mathbf{N}^{\circ}$ de alumnas \\
\hline Si & 130 \\
No & 430 \\
\hline
\end{tabular}

En relación a los métodos anticonceptivos observamos que un gran porcentaje conoce el método del ritmo, el cual no es tan recomendable, ya que el ciclo menstrual no está definido en las adolescentes; seguido del grupo de adolescentes que conocen todos los métodos.

Del total de las encuestadas que iniciaron sus relaciones sexuales, en un escaso porcentaje utilizan métodos anticonceptivos. Reflejándose que se encuentran en un alto riesgo de embarazo precoz, pero en relación con el cuadro anterior refieren que conocen pero no utilizan

Tabla 3. Métodos de planificación familiar que conoce.

\begin{tabular}{lc}
\hline Método & $\mathbf{N}^{\circ}$ de alumnas \\
\hline Método del ritmo & 170 \\
Inyectables & 50 \\
Ritmo - ligaduras & 35 \\
Condón-pastillas del dia siguiente & 70 \\
Todos & 150 \\
No sabe & 80 \\
\hline
\end{tabular}

El mayor porcentaje de estudiantes tienen la autoestima elevada, siendo un mínimo porcentaje que refieren sentirse mal desconociendo el motivo o causa el cual nos invita para un próximo trabajo de investigación. 
Lo resaltante en las estudiantes encuestadas es que el mayor porcentaje tiene dentro de sus planes para el futuro ser profesional, y un $15 \%$ no tiene planes, es un porcentaje preocupante (94 estudiantes), ya que dentro de las encuestadas se tiene estudiantes del quinto año de secundaria.

\section{DISCUSION}

En América Latina, la edad promedio de inicio de actividad sexual es 17 años para la mujer y 15 años para el varón. El $90 \%$ de los varones a los 19 años ya han tenido experiencia sexual; a esa misma edad el $45 \%$ al $65 \%$ de las mujeres.

El iniciar la actividad sexual antes de los 18 años es una decisión sobre la cual influyen e interactúan una serie de factores, (2), en el estudio podemos observar que la edad de la población encuestada está conformada por adolescentes del sexo femenino siendo en su mayoría de 15, 16 y 17 años, quienes cursan el tercero, cuarto y quinto de secundaria

Considerando el inicio de las relaciones sexuales precoz como un problema por el riesgo a los embarazos no deseados, las enfermedades de transmisión sexual y otros, en la investigación se encontró que el 22,9 \% de las estudiantes del colegio "Mariscal Castilla" ya iniciaron sus relaciones sexuales convirtiéndose en un riesgo para los embarazos no deseados.

Se pudo observar que las relaciones sexuales de las adolescente en un mayor porcentaje son aceptadas, seguido por obligación, dinero y un mínimo porcentaje son forzadas, invitándonos a trabajar el tema de autoestima y valores.

Cada año 15 millones de adolescentes dan a luz en el mundo (3), cifra que representa poco más del 10 por ciento del total de nacimientos, informa el Fondo de las Naciones Unidas para la Infancia (UNICEF) a la vez que señala que el embarazo temprano es la principal causa de muerte en mujeres de entre 15 y 19 años de edad. Del total de estudiantes encuestadas en un mínimo porcentaje contestaron que si se embarazaron alguna vez, nos da a conocer que un buen porcentaje se encuentra en riesgo de embarazo debido al inicio precoz de relaciones sexuales.
Como discusión final, realizando el contraste con el fundamento teórico y los antecedentes de trabajos de investigación tendientes a los mismos objetivos; además induciendo a partir del resultado de las encuestas realizadas podemos indicar que las adolescentes de la población estudiada, han llegado a tener embarazo precoz (considerando que las estudiantes son en su mayoría adolescentes) en una proporción de aproximadamente $2 \%$, que a nuestro criterio es un porcentaje elevado siendo el objetivo el $0 \%$.

Además se observa que el entorno social al que está referido la hipótesis de éste trabajo nos indica que los adolescentes interactúan solamente con sus familiares y que de los aspectos sexuales, en mayor proporción lo realizan en el colegio, viéndose además la poca intervención del sector salud. Como una de las necesidades del individuo (adolescentes) en un medio social tiene como requerimientos básicos el de satisfacer sus necesidades primarias dentro de ellos alimentación, vivienda, vestido, implica responder inmediatamente a satisfacer las necesidades requeridas, del total de encuestadas el $23 \%$ han tenido relaciones sexuales y de éste porcentaje el $6,25 \%$ lo han hecho por dinero.

\section{REFERENCIAS BIBLIOGRÁFICAS}

1. Stern C. El embarazo en la adolescencia como problema público: una visión crítica.Salud Publica México.1997;39:137-143.

2. OPS. La Salud Adolescente y el Joven en las Américas. Publicación Científica N. 485. OMS/FNUAP/UNICEF; 1999.

3. UNESCO. Embarazo en adolescentes en América Latina. Enlace. Caracas. Publicación No 13; 1985.

4. Oficina Central de Información. Estadísticas de embarazo en adolescentes. Caracas; 1990.

5. Sing S, Wuef D. Adolescentes de hoy, padres del mañana. Perfil de las Américas. Washington. The Alan Zuhmecher Institute; 1990.

E-mail: paredesmagda@yahoo.com 